\title{
Investigation of Requirements Interdependencies in Existing Techniques of Requirements Prioritization
}

\author{
Fadhl HUJAINAH, Rohani Binti ABU BAKAR, Mansoor ABDULLATEEF ABDULGABBER
}

\begin{abstract}
Requirements prioritization (RP) is considered as a key role in producing a successful system by selecting the most important requirements to be released. Requirements interdependencies (RI) is one of the crucial aspects that need to be addressed in RP, since most of the requirements in reality are not independent and have dependencies between each other. Thus, ignoring RI in RP process may lead to produce inaccurate prioritization result which directly impacts the system's success. In spite of this, little is known about the impact of RI, and obviously further research is urgently required to measure the RI in the RP techniques. Hence, this study aims to investigate and analyze the existence and the execution steps of handling RI in the existing RP techniques to improve the performance of techniques in generating accurate result and assist the researchers and practitioners to select the appropriate technique that can handle RI in prioritization process. The findings indicate that, out of 65 techniques, there are only 4 techniques that handle the RI. The result reveals that these four techniques still suffer from issues of manual process and heavily rely on the experts' participation. Proposing a new technique is recommended to overcome the identified limitations.
\end{abstract}

Keywords: prioritization; requirements; requirements interdependencies

\section{INTRODUCTION}

System development life cycle is constructed with the various phases to be used in order to develop a good quality system [1-3]. Requirement elicitation is an important phase of system development life cycle, since it is the phase where the requirements are elicited and extracted from the stakeholders [4-6] Implementing and developing all the elicited requirements is extremely difficult with limited resources such as budget constraints, short schedule, and limited staff power [7, 8]. On the other hand, the implementation of the stakeholders' requirements is considered as a main factor that can lead to develop and deliver a successful system [4, 9-11]. Prioritizing the requirements is considered as one of the solutions in such a case, since requirements prioritization (RP) is defined as a process of prioritizing the requirements based on the stakeholders' preferences in order to identify the most important requirements among others and to produce a ranked list of requirements [12-14] This requirement list is used in release planning and implementation of the system. Hence, RP is one of the key aspects in producing a successful system that meets the stakeholders' needs [4, $15]$.

Various techniques have been proposed to perform the prioritization process such as AHP [16], PHandler [4], and Dot Voting techniques [17] etc. The major issue that needs to be considered in executing RP is the existence of the dependencies among the requirements, since requirements are not considered as separated segments and requirements are $80 \%$ interdependent and affect each other [18, 19]. These dependencies contribute to the complexity issue in prioritizing the requirements for project release plan [13, 19-21]. For instance, in order to implement requirement B, requirement $A$ must be implemented as it is a prerequisite of requirement $\mathrm{B}$. This implies that ignoring requirement dependencies in the RP process will give rise to a conflict during the implementation release planning of the requirements which is executed based on the produced prioritized list of requirement. There will be possibilities of having high priority requirements that have some prerequisite requirements at a lower priority, so it will be difficult to implement these high priority requirements first unless the prerequisite requirements of a lower priority should be considered and developed $[19,22]$. Despite this, not many researches have focused on the impact of requirements interdependencies in prioritizing the requirements. Further research is needed to measure the existence and execution steps of addressing the RI in the existing RP techniques [19, 20]. Therefore, the overall aim of this study is to investigate the existence of the requirements dependencies in the existing techniques that are available in performing the RP. In addition, the study will investigate how the dependencies between requirements are handled in these techniques.

The rest of this paper is organized as follows: Section 2 explains the research methodology which is used to conduct this study. Section 3 elaborates and discusses the findings of this study, while section 4 highlights the conclusion along with future recommendation based on the identified issues.

\section{RESEARCH METHOD}

To achieve the defined aim of this research, a research method was constructed as shown in Fig. 1. It is comprised of 4 phases: research questions, search process, related studies selection, and analysis of the selected studies. The research activities began with research questions phase, where the scope of this study is defined by formulating the research questions based on the identified objective of this research. The objective of this study is to analyze, review and investigate the requirement interdependencies in existing requirements prioritization techniques. To obtain the defined objective, two research questions were formulated as follows:

- RQ1: What are the existing techniques of RP that handle the requirement interdependencies in prioritizing the requirements?

- RQ2: How do the current RP techniques handle RI in the prioritization process?

After specifying the research questions, search process phase was executed by searching for the possible related existing studies. The related existing studies were elicited and collected based on the specified research questions 
from the published research studies of conferences, IEEE Bulletins, journals, workshops, books chapters. Relevant and well-known electronic resources used for extracting the related studies include IEEE Computer Society Digital Library, the Springer link, ISI Web of Knowledge, the Springer link, ACM and Science Direct, Google Scholar $[12,23,24]$. In addition, list of keywords was formulated based on the research questions to search for related research studies. The list of keywords used was employed with combination of Boolean operators (AND, OR) in order to extend the searching process. The formulated lists of keywords used are as follows:

- Requirements Prioritization (OR, AND) selection.

- Requirements interdependencies OR dependency.

- Requirements interdependencies in Requirements prioritization.

- Requirements prioritization techniques OR methods OR frameworks, OR approaches OR models.

- Requirements interdependencies (OR) dependency in requirements prioritization techniques OR methods OR frameworks, OR approaches OR models.

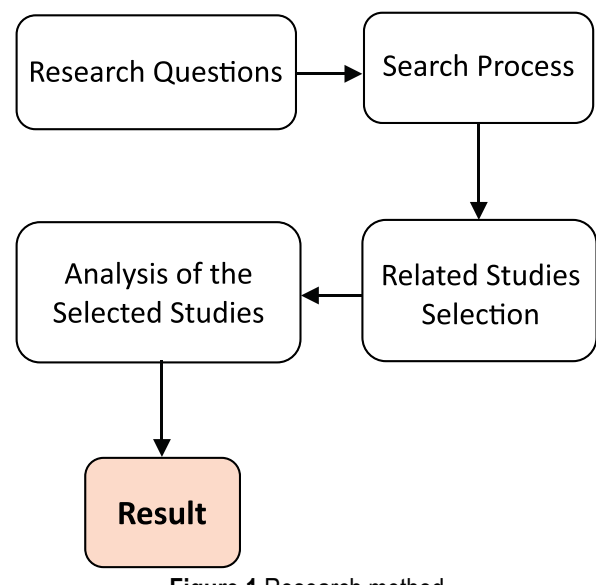

Figure 1 Research method

Furthermore, the study selection phase was executed based on the identified research questions. The title, abstract and content of each collected study was critically studied to select only the studies that can at least provide potential answer to the research questions. Thus, 52 studies were selected as most relevant studies based on the defined research questions that are capable to address the listed research questions. Finally, analysis of the selected studies was performed to analyze the findings and result of the study. The selected relevant studies have been analyzed critically to answer the formulated research questions.

\section{FINDINGS AND DISCUSSION}

To address the specified research questions as mentioned in previous section, each prioritization technique was identified and analyzed critically in terms of addressing the RI and how RI was handled in the prioritizing process. Tab. 1 presents the outcome result of the analysis of all RP techniques ( 65 techniques) that were identified from the selected studies. Based on the derived result, most of the RP techniques did not address the RI in their prioritization processes due to the fact that majority of the techniques were proposed with the aim of prioritizing requirements and assuming that the requirements are free of dependencies. However, most of the RP studies highlight the requirements interdependencies as a required future enhancement in their research. Thus, only 4 out of 65 RP techniques handled the RI in their prioritization processes. These techniques are Multi-Aspects Based RP [25], Value Based Requirements Prioritization [26], Mathematical Programming [19], and Interactive Requirements Prioritization techniques [27].

The aim of Multi-Aspects Based Requirement Prioritization technique was to prioritize the requirements based on the business aspects [25]. In this technique, RI was considered as one of the business aspects (dependency aspect), where the technique allows the stakeholders to assign a value of the dependency to each requirement individually. However, handling RI in this way is poor, since the dependencies between the requirements have to be taken based on the dependency rate of the requirement on others instead of letting the stakeholders directly give a value of the dependency to each requirement separately without considering the possible dependencies of the requirements among each other [27].

On the other hand, the Value Based Requirements Prioritization technique was reported in [26]. This technique addresses the dependencies among requirements in the requirements selection process as compared to other techniques. The dependencies are addressed using comma separated lists of requirements. The way to address RI in these techniques is considered as poor handling with large set of requirements, since it will be hard to fill in the dependencies between the requirements. In addition, the inability to sort hierarchically the requirements is reported as another issue in this technique.

Mathematical Programming technique was proposed to perform the RP process with addressing the dependencies between the requirements [19]. In this technique, the dependency was identified by randomly generating the dependency ratio between the requirements with the condition that the requirement cannot be selected and released unless its predecessor is selected prior to it. In conclusion, more effort is needed in order to evaluate the technique's way of addressing the RI and generalize the result. Also, the difficulties of pointing out the priority value of each requirement is identified as another issue in this technique [19].

In [27], another technique named "Interactive Requirements Prioritization" was presented to prioritize the requirements with considering the dependencies between them. The analyst (who elicited these requirements) provides the list of the requirements along with the classification of each requirement in three priority groups: high, medium, low. The priority rank is induced by the dependencies between requirements. For example, the requirements in the high rank priority group should precede those requirements that are in medium priority group. Although this technique provided such a good solution of handling RI during prioritizing the requirements compared to other techniques, the participation of the highly professional analyst is required to conduct the process. Also there is a need to conduct more case studies to evaluate its effectiveness [27]. 
Table 1 Analysis of the existing techniques of requirements prioritization

Technique Name

\begin{tabular}{|c|c|c|c|}
\hline No & Technique Name & Year & Handle RI \\
\hline 1 & A Clustering Based Technique For Large Scale Prioritization [7] & 2014 & $x$ \\
\hline 2 & A Conceptual Model And Process For Client-Driven Agile RP [17] & 2010 & $x$ \\
\hline 3 & A Preference Weights Model For Prioritizing Software Requirements [14] & 2014 & $x$ \\
\hline 4 & A Web-Based Multi-Criteria Decision Making Tool For Software RP [15] & 2014 & $x$ \\
\hline 5 & Analytic Hierarchy Process (AHP) [16] & 1998 & $x$ \\
\hline 6 & Architecture Trade-off Analysis Method (ATAM) [28] & 2002 & $x$ \\
\hline 7 & Attributed Goal Oriented Requirements Analysis [13] & 2002 & $x$ \\
\hline 8 & Benefit and Cost Prediction [13] & 2008 & $x$ \\
\hline 9 & Binary Priority List $[7,15]$ & 2010 & $x$ \\
\hline 10 & Binary Search Tree [16] & 1998 & $x$ \\
\hline 11 & Bubble Sort [16] & 1998 & $x$ \\
\hline 12 & Case Based Ranking [29] & 2013 & $x$ \\
\hline 13 & Cognitive Driven Requirement Prioritization [13] & 2010 & $x$ \\
\hline 14 & Conceptual Model of Agile Requirement Prioritization [17] & 2008 & $x$ \\
\hline 15 & Correlation-Based Priority Assessment Framework [30] & 2006 & $x$ \\
\hline 16 & Cost- Value Approach [13] & 1997 & $x$ \\
\hline 17 & Cost-Benefit Analysis Method [28] & 2002 & $x$ \\
\hline 18 & Cumulative Voting Or 100 Dollars [4] & 2000 & $x$ \\
\hline 19 & Dot Voting $[17,31]$ & 2007 & $x$ \\
\hline 20 & Eclipse Process Framework [17] & 2008 & $x$ \\
\hline 21 & Evolve [13] & 2004 & $x$ \\
\hline 22 & Fuzzy AHP [13] & 2002 & $x$ \\
\hline 23 & Fuzzy Hierarchical Cumulative Voting [32] & 2014 & $x$ \\
\hline 24 & Hierarchical Cumulative Voting (HCV) $[4,8]$ & 2006 & $x$ \\
\hline 25 & Hierarchy AHP [16] & 1998 & $x$ \\
\hline 26 & Interactive Requirements Prioritization [27] & 2013 & $\checkmark$ \\
\hline 27 & Kano Model $[17,31]$ & 2007 & $x$ \\
\hline 28 & Lanchester Theory [13] & 2008 & $x$ \\
\hline 29 & Mathematical Programming Technique [19] & 2010 & $\checkmark$ \\
\hline 30 & Moscow [31] & 2007 & $x$ \\
\hline 31 & Minimal Spanning Tree [16] & 1998 & $x$ \\
\hline 32 & Multi-Aspects Based RP [25] & 2014 & $\checkmark$ \\
\hline 33 & Multi-Criteria Preference Analysis Requirements Negotiation [13] & 2002 & $x$ \\
\hline 34 & Multi-Objective Next Release Problem [21, 27] & 2007 & $x$ \\
\hline 35 & Multi-Voting System [31] & 2006 & $x$ \\
\hline 36 & Numerical Assignment [31] & 1996 & $x$ \\
\hline 37 & Pair-Wise Comparison [33] & 1996 & $x$ \\
\hline 38 & Partial Order Assimilation Approach [30] & 2014 & $x$ \\
\hline 39 & PHandler [4] & 2015 & $x$ \\
\hline 40 & Ping Pong Balls [17] & 2004 & $x$ \\
\hline 41 & Planguage [29] & 2005 & $x$ \\
\hline 42 & Planning Game [33] & 1999 & $x$ \\
\hline 43 & Planning Game Combined With AHP (PGCAHP) [33] & 2004 & $x$ \\
\hline 44 & Priority Groups [16] & 1998 & $x$ \\
\hline 45 & Quality Functional Deployment $[29,31]$ & 1990 & $x$ \\
\hline 46 & Ranking [16] & 1998 & $x$ \\
\hline 47 & Ranking Based On Product Definition $[17,31]$ & 2002 & $x$ \\
\hline 48 & Relative Weighting [17] & 2005 & $x$ \\
\hline 49 & Requirement Triage [34] & 2009 & $x$ \\
\hline 50 & Requirement Uncertainty Prioritization Approach [35] & 2012 & $x$ \\
\hline 51 & Round the Group Prioritization $[17,31]$ & 2006 & $x$ \\
\hline 52 & Software Engineering Risk: Understanding And Management (SERUM) [28] & 1999 & $x$ \\
\hline 53 & Simple Multi-Criteria Rating Technique by Swing [36] & 2004 & $x$ \\
\hline 54 & Software Architecture Analysis Method (SAAM) [28] & 2002 & $x$ \\
\hline 55 & StakeRare [8] & 2012 & $x$ \\
\hline 56 & Theme Screening/Scoring [17] & 2005 & $x$ \\
\hline 57 & Top Ten $[4,13]$ & 2002 & $x$ \\
\hline 58 & Two-Step Value-Based Requirements Prioritization Approach Based on TOPSIS [26] & 2013 & $x$ \\
\hline 59 & Using the Fuzzy Multi-Criteria Decision Making Approach for Software RP [13] & 2015 & $x$ \\
\hline 60 & Value Based Requirements Prioritization [26] & 2013 & $\checkmark$ \\
\hline 61 & Value Based Intelligent Requirement Prioritization [4] & 2011 & $x$ \\
\hline 62 & Value Oriented Requirements Prioritization [28] & 2007 & $x$ \\
\hline 63 & Weighted Criteria Analysis $[17,31]$ & 2005 & $x$ \\
\hline 64 & Wiegers' Matrix Approach [31] & 1999 & $x$ \\
\hline 65 & Winwin $[8,13]$ & 2000 & $x$ \\
\hline
\end{tabular}




\section{CONCLUSION}

$\mathrm{RI}$ is a key aspect in requirements prioritization, since prioritizing the requirements without handling the dependencies among them will impact the quality of the prioritization result. This study provided a comprehensive investigation of RI in the existing RP techniques in terms of measuring the RI existence in current techniques along with presenting the execution steps that are used to handle RI in their prioritization process. This will assist the researchers and practitioners to identify and select the suitable technique in handling the RI and help them to enhance the quality performance of the current techniques.

Moreover, this study was conducted based on the research method which consisted of 4 activities: research questions, search process, related studies selection, and analysis of the selected studies. The study's findings reveal that most of the current RP techniques lack handling the requirements interdependencies in the prioritization process. Out of 65 RP techniques, only 4 handle the RI. These techniques are Interactive Requirements Prioritization, Multi-Aspects Based RP, Mathematical Programming, and Value Based Requirements Prioritization techniques. As revealed from the findings, these four techniques have limitations in terms of manual process and the need of the experts' participation. Also, more work is needed to assess the effectiveness of these techniques in terms of evaluating and verifying the capability of their process in addressing the requirements interdependencies. Thus, a new technique is recommended to be proposed in order to handle the RI automatically with reducing the experts' involvement especially with the large set of requirements.

\section{Acknowledgements}

The authors appreciate the efforts of the Ministry of Education Malaysia, University Malaysia Pahang (UMP) and Ministry of Higher Education Yemen for supporting this research. This work is supported in part by the Fundamental Research Grant: A New Model for Automated Stakeholder Quantification and Prioritization Based on User Needs for Software System Development from the Ministry of Education Malaysia under Grant RDU190164 and the PGRS170393 Grant from UMP.

\section{REFERENCES}

[1] Yilmaz, M. \& Connor, R. V. O. (2016). A Scrumban integrated gamification approach to guide software process improvement: a Turkish case study. Tehnicki vjesnik, 23(1), 237-245. https://doi.org/10.17559/TV-20140922220409

[2] Huđek, M., Savić, Z., \& Radović, D. (2017). Modeling a business intelligence system for school management on local government level. Tehnicki vjesnik, 24(3), 821-828. https://doi.org/10.17559/TV-20160205121415

[3] Crawford, B., Soto, R., Johnson, F., Misra, S., Paredes, F., \& Olguín, E. (2015). Software Project Scheduling using the Hyper-Cube Ant Colony Optimization algorithm. Tehnicki vjesnik, 22(5). https://doi.org/10.17559/TV-20140519212813

[4] Babar, M. I., Ghazali, M., Jawawi, D. N. A., Shamsuddin, S. M., \& Ibrahim, N. (2015). PHandler: an expert system for a scalable software requirements prioritization process. Knowledge-Based Systems, 84, 179-202. https://doi.org/10.1016/j.knosys.2015.04.010
[5] Hujainah, F., Al-Haimi, B., Nasser, A. B., Hujainah, A., \& Al-Bashiri, H. (2017). Applying architectural analysis for current software systems: A case study of KFC and pizza hut online food ordering systems in Malaysia. Advanced Science Letters, 23(9), 9145-9151. https://doi.org/10.1166/asl.2017.10042

[6] Hujainah, F., Dahlan, H., Al-Haimi, B., Hujainah, A., AlBashiri, H., \& Abdulgabber, M. A. (2016). New usability guidelines with implementation ways of mobile learning application based on mobile learning usability attributes. Indian Journal of Science and Technology, 9(37). https://doi.org/10.17485/ijst/2016/v9i37/94323

[7] Achimugu, P., Selamat, A., \& Ibrahim, R. (2014). A clustering based technique for large scale prioritization during requirements elicitation. In Herawan T., Ghazali R., Deris M. (eds) Recent Advances on Soft Computing and Data Mining. Advances in Intelligent Systems and Computing, Springer, Cham, 287, 30-39. https://doi.org/10.1007/978-3-319-07692-8

[8] Lim, S. L. \& Finkelstein, A. (2012). StakeRare : using social networks and collaborative filtering for large-scale requirements eliciation. IEEE Transactions on Software Engineering, 38, 707-735. https://doi.org/10.1109/TSE.2011.36

[9] Hujainah, F., Bakar, R. B. A., \& Abdulgabber, M. A. (2019). StakeQP: A semi-automated stakeholder quantification and prioritisation technique for requirement selection in software system projects. Decision Support Systems, 121, 94-108. https://doi.org/10.1016/j.dss.2019.04.009

[10] Alhroob, E. \& Ghani, N. A. (2018). Fuzzy Min-Max Classifier Based on New Membership Function for Pattern Classification: A Conceptual Solution. The $8^{\text {th }}$ IEEE International Conference on Control System, Computing and Engineering (ICCSCE), 131-135. https://doi.org/10.1109/ICCSCE.2018.8685029

[11] Hujainah, F., Dahlan, H., \& Al-haimi, B. (2013). Usability Guidelines of Mobile Learning Application. Journal of Information Systems Research and Innovation, 5(2), 70-77.

[12] Hujainah, F., Abu Bakar, R. B., Al-haimi, B., \& Abdulgabber, M. A. (2018). Stakeholder quantification and prioritisation research: A systematic literature review. Information and Software Technology, 102, 85-99. https://doi.org/10.1016/j.infsof.2018.05.008

[13] Hujainah, F., Bakar, R. B. A., Abdulgabber, M. A., \& Zamli, K. Z. (2018). Software Requirements Prioritisation: A Systematic Literature Review on Significance, Stakeholders, Techniques and Challenges. IEEE Access, 6, 71497-71523. https://doi.org/10.1109/ACCESS.2018.2881755

[14] Achimugu, P., Selamat, A., \& Ibrahim, R. (2014). A preference weights model for prioritizing software requirements. Hwang, D., Jung, J. J., \& Nguyen, N. T. (eds) Computational Collective Intelligence. Technologies and Applications. ICCCI 2014. Lecture Notes in Computer Science, Springer, Cham, 8733, 30-39. https://doi.org/10.1007/978-3-319-11289-3_4

[15] Achimugu, P., Selamat, A., \& Ibrahim, R. (2014). A webbased multi-criteria decision making tool for software requirements prioritization. Hwang D., Jung J. J., \& Nguyen N. T. (eds) Computational Collective Intelligence. Technologies and Applications. ICCCI 2014. Lecture Notes in Computer Science, Springer, Cham, 8733, 444-453. https://doi.org/10.1007/978-3-319-11289-3_45

[16] Karlsson, J., Wohlin, C., \& Regnell, B. (1998). An evaluation of methods for prioritizing software requirements. Information and Software Technology, 39, 939-947. https://doi.org/10.1016/S0950-5849(97)00053-0

[17] Racheva, Z., Daneva, M., Herrmann, A., \& Wieringa, R. J. (2010). A conceptual model and process for client-driven agile requirements prioritization. The Fourth International Conference on Research Challenges in Information Science 
(RCIS), 287-298. https://doi.org/10.1109/RCIS.2010.5507388

[18] Carlshamre, P., Sandahl, K., Lindvall, M., Regnell, B., \& Natt och Dag, J. (2001). An industrial survey of requirements interdependencies in software product release planning. Proceedings of the IEEE International Conference on Requirements Engineering, 84-91. https://doi.org/10.1109/ISRE.2001.948547

[19] Li, C., van den Akker, M., Brinkkemper, S., \& Diepen, G. (2010). An integrated approach for requirement selection and scheduling in software release planning. Requirements Engineering, 15(4), 375-396. https://doi.org/10.1007/978-3-540-73031-6_7

[20] Carlshamre, P. \& Regnell, B. (2000). Requirements lifecycle management and release planning in market-driven requirements engineering processes. The $11^{\text {th }}$ International Workshop on Database and Expert Systems Applications., (September), 961-965. https://doi.org/10.1109/DEXA.2000.875142

[21] Zhang, Y., Harman, M., \& Mansouri, S. (2007). The multiobjective next release problem. ACM Genetic and Evolutionary Computation Conference, 1129-1136. https://doi.org/10.1145/1276958.1277179

[22] Hujainah, F., Bakar, R. B. A., Al-Haimi, B., \& Abdulgabber, M. A. (2018). Investigation of Stakeholder Analysis in Requirement Prioritization Techniques. Advanced Science Letters, 24(10), 7227-7231. https://doi.org/10.1166/asl.2018.12919

[23] Alhroob, E., Mohammed, M. F., Lim, C. P., \& Tao, H. (2019). A Critical Review on Selected Fuzzy Min-Max Neural Networks and Their Significance and Challenges in Pattern Classification. IEEE Access, 7, 56129-56146. https://doi.org/10.1109/access.2019.2911955

[24] Al Sayaydeh, O. N., Mohammed, M. F., \& Lim, C. P. (2019). Survey of Fuzzy Min-Max Neural Network for Pattern Classification Variants and Applications. IEEE Transactions on Fuzzy Systems, 27(4), 635-645. https://doi.org/10.1109/TFUZZ.2018.2865950

[25] Sher, F., Jawawi, D. N. A., Mohamad, R., \& Babar, M. I. (2014). Multi-aspects based requirements priortization technique for value-based software developments. In Proceedings - 2014 International Conference on Emerging Technologies, ICET 2014. 1-6. https://doi.org/10.1109/ICET.2014.7021007

[26] Kukreja, N., Payyavula, S. S., Boehm, B., \& Padmanabhuni, S. (2013). Value-based requirements prioritization: usage experiences. Procedia Computer Science, 16, 806-813. https://doi.org/10.1016/j.procs.2013.01.084

[27] Tonella, P., Susi, A., \& Palma, F. (2013). Interactive requirements prioritization using a genetic algorithm. Information and Software Technology, 55(1), 173-187. https://doi.org/10.1016/j.infsof.2012.07.003

[28] Clements, P., Kazman, R., \& Klein, M. (2002). Evaluating software architectures: methods and case studies. ( $1^{\text {st }}$ Ed.). Addison-Wesley Longman Publishing Co., Inc. Boston, MA, USA.

[29] Perini, A., Susi, A., \& Avesani, P. (2013). A machine learning approach to software requirements prioritization. IEEE Transactions on Software Engineering, 39(4), 445461. https://doi.org/10.1109/TSE.2012.52

[30] Easmin, R., Ul Gias, A., \& Khaled, S. M. (2014). A partial order assimilation approach for software requirements prioritization. International Conference on Informatics, Electronics and Vision, ICIEV 2014, 1-5. https://doi.org/10.1109/ICIEV.2014.6850776

[31] Racheva, Z., Daneva, M., \& Buglione, L. (2008). Supporting the dynamic reprioritization of requirements in agile development of software products. Software Product Management, IWSPM'08, The Second International Workshop on, IEEE, (I), 49-58. https://doi.org/10.1109/IWSPM.2008.7
[32] Sharif, N., Zafar, K., \& Zyad, W. (2014). Optimization of requirement prioritization using computational intelligence technique. International Conference on Robotics and Emerging Allied Technologies in Engineering (iCREATE 2014), 228-234. https://doi.org/10.1109/iCREATE.2014.6828370

[33] Karlsson1, L., Berander, P., Regnell, B., \& Wohlin, C. (2004). Requirements prioritisation: an experiment on exhaustive pair-wise comparisons versus planning game partitioning. Proceedings of the $8^{\text {th }}$ Conference on Empirical Assessment in Software Engineering, Edinburgh, UK, 145154. https://doi.org/10.1049/ic:20040407

[34] Duan, C., Laurent, P., Cleland-Huang, J., \& Kwiatkowski, C. (2009). Towards automated requirements prioritization and triage. Requirements Engineering, 14(2), 73-89. https://doi.org/10.1007/s00766-009-0079-7

[35] Voola, P. \& Babu, A. V. (2012). Requirements uncertainty prioritization approach: a novel approach for requirements prioritization. Softw Eng Int J (SEIJ), 2(2), 37-49.

[36] Avesani, P., Bazzanella, C., Perini, A., \& Susi, A. (2004). Supporting the requirements prioritization process. A machine learning approach. Proceedings of the $16^{\text {th }}$ International Conference on Software Engineering and Knowledge Engineering (SEKE 2004), KSI Press, Banff, Alberta, Canada, 2004, 306-311.

\section{Contact information:}

Fadhl HUJAINAH, Doctor of Philosophy (Software Engineering) Software Engineering Research Group,

Faculty of Computer Systems and Software Engineering, Universiti Malaysia Pahang, University Malaysia Pahang, Lebuhraya Tun Razak, Kuantan 26300, Malaysia fadelhogina@gmail.com

Rohani Binti ABU BAKAR, Associate Professor Dr. Soft Computing and Intelligent System Research Group, Faculty of Computer Systems and Software Engineering, Universiti Malaysia Pahang, University Malaysia Pahang, Lebuhraya Tun Razak, Kuantan 26300, Malaysia rohani@ump.edu.my

Mansoor Abdullateef ABDULGABBER, Assistant Professor Dr. Faculty of Computer and Cyber Science, Software Engineering, University Prince Mugrin,

Al-Madinah 41499, Saudi Arabia

h.mansoor@upm.edu.sa 